Recepción: 31/ 03/ 2016

Aceptación: 28 / 06/ 2016

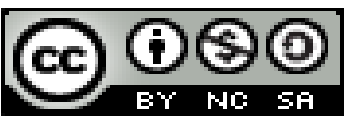

Ciencias de la salud

Publicación: 06/ 01/2017

Articulo Original

\title{
La evaluación de la satisfacción en salud: un reto en la atención estomatológica
}

The evaluation of health satisfaction: a challenge in stomatological care

A avaliação da satisfação em saúde: um desafio no atendimento odontológico

\author{
Maritza Berenguer-Gouarnaluses ${ }^{\mathrm{I}}$ \\ maritzab@medired.scu.sld.cu \\ Nuria Rosa Medianeja Álvarez II \\ nuria.rosaa@gmail.com \\ Marlene Montoya Medina III \\ mmmarlene@gmail.com
}

Correspondencia: maritzab@medired.scu.sld.cu

I. Médico Universidad de Ciencias Médicas, Facultad de Estomatología, Santiago de Cuba, Cuba.

II. Policlínico Universitario Josué País García, Santiago de Cuba, Cuba.

III. Médico Universidad de Ciencias Médicas, Santiago de Cuba, Cuba. 


\section{Resumen}

Con el desarrollo de la humanidad, y en particular con los avances en el terreno de la medicina, es necesario hacer un llamado para humanizar la asistencia en salud y ofrecer servicios de mayor calidad. La calidad en la atención médica debe estar basada en actividades encaminadas a garantizar los servicios de salud accesibles y equitativos con profesionales óptimos y teniendo en cuenta los recursos disponibles, logrando la satisfacción del usuario con la atención recibida. El presente trabajo es un estudio descriptivo y transversal, con el objetivo de evaluar el resultado mediante la determinación de niveles de satisfacción de los proveedores de salud bucal, con la atención que brindan y de los usuarios con el servicio recibido nivel de satisfacción de los estomatólogos generales y licenciados en atención estomatológica a la población preescolar y escolar del reparto Abel Santamaría , y usuarios en el período comprendido de enero - diciembre del 2015, mediante la metodología propuesta en el documento para este fin. El universo estuvo constituido por el total de Especialistas de Estomatología General Integral que se encontraban laborando $(\mathrm{N}=2)$, Licenciadas en Atención Estomatológica $(\mathrm{N}=3)$, que realizan la actividad preventiva, y usuarios una muestra de 148 escolares y 47 pre-escolares, para la evaluación de la satisfacción profesional. Se evaluó la satisfacción como dimensión de la calidad mediante criterios, indicadores y estándares utilizados, el cual fue adecuado.

Palabras clave: Calidad, evaluación, satisfacción.

\section{Abstract}

With the development of humanity, and in particular with advances in the field of medicine, it is necessary to make a call to humanize health care and offer higher quality services. Quality in health care should be based on activities aimed at ensuring accessible and equitable health services with optimal professionals and taking into account available resources, achieving user satisfaction with the care received. The present work is a descriptive and transversal study, with the objective of evaluating the result by means of the determination of levels of satisfaction of the oral health providers, with the care they provide and of the users with the service received level of satisfaction of the stomatologists General and graduates in stomatological care to the preschool and school population of the Abel Santamaría cast, and users in the period from January to December 2015, using the methodology proposed in the document for this purpose. The universe 
was constituted by a total of Specialists of General Integral Stomatology who were working $(\mathrm{N}=$ 2), Graduates in Stomatological Care $(\mathrm{N}=3)$, who perform preventive activity, and users a sample of 148 students and 47 pre- School, for the evaluation of professional satisfaction. Satisfaction was assessed as a dimension of quality through criteria, indicators and standards used, which was adequate.

Key words: Evaluation, quality, satisfaction.

\section{Resumo}

Com o desenvolvimento da humanidade, especialmente com os avanços no campo da medicina, é necessário chamar a humanizar os cuidados de saúde e prestação de serviços de maior qualidade. Cuidados de saúde de qualidade deve basear-se em esforços para garantir serviços acessíveis e equitativos profissionais de saúde e óptima, tendo em conta os recursos disponíveis, alcançar a satisfação do usuário com o atendimento recebido. Este trabalho é um estudo descritivo, com o objetivo de avaliar o resultado pela determinação dos níveis de satisfação entre os provedores de saúde bucal, com assistência prestada e os usuários com o atendimento recebido nível de odontologia satisfação graduados em cuidados gerais e odontológica à pré-escola e em idade escolar população elenco Abel Santamaria, e os usuários no período de janeiro a dezembro de 2015, utilizando a metodologia proposta no documento para essa finalidade. O universo foi constituído pelo total de Especialistas Integral General Dentistry que estavam trabalhando $(\mathrm{N}=$ 2), licenciado em atenção Estomatologia $(\mathrm{N}=3)$, que executar a atividade preventiva e usuários com uma amostra de 148 alunos e 47 pré escola, para a avaliação da satisfação no trabalho. Foi avaliada a satisfação como uma dimensão da qualidade através de critérios, indicadores e padrões utilizados, que foi adequada.

Palavras chave: Qualidade; avaliação; satisfação.

\section{Introducción}

En las últimas décadas del siglo XX las transformaciones sociales y económicas han producido cambios en la salud de la población y, por tanto, en las políticas de prestación de salud a escala mundial. En tales condiciones se promueve una atención de alta calidad centrada en el usuario o cliente y profesional. ${ }^{1}$

Para evaluar la calidad se han utilizado varias herramientas, que incluyen: monitoreos, modelos, instrumentos y propuestas metodológicas como la de Jiménez Cangas L [et al] 1996, de la 
Escuela Nacional de Salud Pública de Cuba, la cual se confeccionó para la atención primaria y es utilizada para todos los niveles. ${ }^{2}$

Según Laurenza y Marrazino, el monitoreo constituye una herramienta de evaluación sistemática de procesos y resultados. Esta fue utilizada en una investigación en hospitales públicos de Argentina, donde se aplicó el programa PICAM-Baires, y constituyó una novedosa experiencia de monitoreo externo. ${ }^{3}$

De la misma manera, son muchas las investigaciones realizadas en este campo, tanto a escala nacional como internacional, pero también de diversos grupos poblacionales, tales como: adulto mayor, atención materna infantil, servicios estomatológicos, adolescentes, prescripciones médicas y programas sanitarios, entre otros, tanto en la atención primaria de salud como en la secundaria ${ }^{4-5}$

La calidad de los servicios de salud en el mundo actual se ha convertido en motivo de interés para todo salubrista, en busca siempre de la obtención de mejores resultados para la población, proporcionando el mínimo de riesgos tanto para el consumidor como para los proveedores de salud, y un adecuado aprovechamiento de los recursos disponibles para ejecutar las acciones. El enfoque sistémico de la calidad permite establecer tres componentes para su estudio: la estructura, el proceso y el resultado; sobre éstos se han trazado los métodos para la evaluación de la calidad ${ }^{6}$.

Las nuevas tendencias en calidad asistencial se enmarcan dentro de la mejora continua, que pretende identificar oportunidades de mejorar utilizando como herramientas fundamentales el rediseño o reingeniería de proceso. La mejora continua no se preocupa por conocer quien ha podido cometer errores, sino de averiguar, que proceso necesita mejorar. En el ejercicio médico, la mejora continua combinará ética, técnica y arte. ${ }^{7}$

Se habla de calidad en la atención médica o calidad asistencial cuando se realizan diferentes actividades encaminadas a garantizar los servicios de salud accesibles y equitativos con profesionales sumamente buenos y teniendo en cuenta los recursos disponibles para lograr la satisfacción del usuario con la atención recibida. 
Se ha partido de considerar la calidad de los servicios de salud como el concepto que refleja la correspondencia de las características de los servicios ofertados a la población con el sistema de normas valorativas explícitas e implícitas que existen en un momento determinado en una sociedad concreta. Se han reconocido las siguientes dimensiones de la calidad: componente científico-técnico, accesibilidad, efectividad, satisfacción y eficiencia. ${ }^{1}$

La calidad posee una connotación histórico-cultural, es específica para una sociedad concreta, para un momento histórico determinado, para una institución en particular, y en ella juegan un importante papel los factores subjetivos o psicosociales (las creencias, la idiosincrasia, los valores, entre otros). La satisfacción de los usuarios y los proveedores de salud constituye una importante dimensión subjetiva del proceso de calidad de la atención que ofrece una institución. ${ }^{8}$

Según Donabedian "el grado de calidad es la medida en que la atención prestada es capaz de alcanzar el equilibrio más favorable entre peligros y bondades" e incluye estructura, procesos y resultados como los tres componentes de una armazón conceptual para evaluar la atención sanitaria y, bajo esta teoría, existe una relación funcional fundamental entre estos tres elementos tal que uno le sigue al otro. ${ }^{1}$

Como definen B. Zas y otros, el proceso de la calidad total llevada a su máxima expresión se convierte en una forma de vida organizacional, hace que cada persona involucrada desempeñe sus tareas completas y cabalmente, en el momento preciso y en el lugar que le corresponde. Esta se define por el usuario y no por la institución; es un enfoque de dar al usuario lo que él necesita, desea, quiere y demanda, es crear usuarios satisfechos. ${ }^{1}$

Aunque satisfacer las expectativas de los usuarios de los servicios de salud constituye un proceso complejo de intersubjetividades, el fin último de la prestación de los servicios de salud no se diferencia de la de otro tipo de servicio: satisfacer a los usuarios (pacientes y acompañantes). Pero sólo se puede satisfacer a los usuarios realmente, si los trabajadores que brindan los servicios también se encuentran satisfechos, y esto hace más compleja aún la trama y las acciones encaminadas por los gestores de estos servicios, para lograr un funcionamiento que cumpla con todas las condiciones necesarias para el logro de una excelencia. ${ }^{1}$ 
Dentro de los indicadores de la calidad que miden resultados, y al cual se le otorga cada vez mayor importancia, se incluye la satisfacción del paciente con la atención recibida, mediante la prestación de un servicio de mayor calidad. ${ }^{1}$

La satisfacción del paciente es la dimensión más estudiada cuando se evalúa calidad, pero a la vez es un indicador que mide resultados, al cual se le otorga cada vez mayor importancia; también se incluye la satisfacción del paciente con la atención recibida y la del prestador con la atención brindada. Esta puede definirse como la medida en que la atención sanitaria y el estado de salud resultante cumplen con las expectativas del usuario. ${ }^{1}$

En ese mismo orden Roger Ruiz-Moral, plantea en su estudio que la satisfacción de los pacientes se considera un importante componente de la calidad de la atención sanitaria pudiéndose medir mediante una amplia variedad de formas y mecanismos. Entre los fines de recoger esta información está alertar a proveedores de servicios sobre preocupaciones, necesidades y percepciones de los usuarios acerca de la atención recibida, pero también son útiles para diseñar programas de planificación, evaluación e identificación de áreas de mejora. Un aspecto del ámbito general de la atención sanitaria que valoran mucho los usuarios es la relación personal con el proveedor, incluso por encima de competencias más técnicas. ${ }^{9}$

La Calidad de la atención estomatológica está vinculada con la forma de valorar el trabajo de nuestra especialidad, con sus cualidades esenciales, caracterizada fundamentalmente por el proceso y los factores que intervienen en la prestación de los servicios, dirigidos a alcanzar buenos resultados y efectos en salud bucal. ${ }^{10}$

\section{Metodología}

Se realizó un estudio de tipo descriptivo transversal para de evaluar el resultado mediante la determinación del nivel de satisfacción de los proveedores y usuarios de salud bucal, perteneciente al Policlínico "Josué País García” del Municipio Santiago de Cuba en el período comprendido de enero a diciembre del 2015.

Para llevar a cabo esta investigación se utilizó la metodología propuesta para estos fines, por la Facultad de Salud Pública de Ciudad de la Habana de Agra Varela Y, y en con su cumplimiento, 
el estudio se realizó en dos etapas: preparación del proceso evaluativo y ejecución del proceso evaluativo. ${ }^{11}$

La población en estudio estuvo constituida por proveedores y usuarios.

\section{Proveedores}

El universo estuvo integrando, por el total de Especialistas de Estomatología General Integral que se encontraban laborando $(\mathrm{N}=2)$, Licenciadas en Atención Estomatológica $(\mathrm{N}=3)$, que realizan la actividad preventiva.

\section{Usuarios}

El universo estuvo integrado por la matrícula de 4to grado de la Escuela "Raúl Gómez García" ( $\mathrm{N}=240)$ y los niños de preescolar del Circulo Infantil "Los Abuelitos”, (N=47).

Se calculó un muestra para la población de 4to grado $(n=148)$, la cual se seleccionó a través de un muestreo aleatorio simple, a través de la tabla de números aleatorios, hasta completar el tamaño calculado.

\section{Resultados y Discusión}

Los resultados obtenidos en la evaluación del nivel de satisfacción de estomatólogos y licenciados con la atención que brindan en la atención primaria, se evaluaron 13 criterios solo el $7,7 \%$ de los criterios resultó adecuado y 12 criterios resultaron inadecuados para un 92,3\%. Con respectos al nivel de satisfacción de los usuarios con la atención recibida, la evaluación fue adecuada con un $100 \%$ de los criterios al estándar establecido.

La satisfacción de los profesionales puede influir en la calidad de los servicios que prestan a la población. 
Tabla 1. Evaluación de la satisfacción de los Estomatólogos y Licenciados en Atención Estomatológica con la atención que brindan.

\begin{tabular}{|c|c|c|c|}
\hline Criterios & $\begin{array}{c}\text { Estándar } \\
\%\end{array}$ & $\begin{array}{c}\text { Resultados } \\
\%\end{array}$ & $\begin{array}{l}\text { Evaluación } \\
\qquad \text { A o I }\end{array}$ \\
\hline 1. Le gusta su trabajo & 90 & 95 & A \\
\hline $\begin{array}{l}\text { 2. Está satisfecho con la calidad del } \\
\text { trabajo que realiza }\end{array}$ & 90 & 60 & I \\
\hline $\begin{array}{l}\text { 3. Preparación científico técnica para su } \\
\text { desempeño }\end{array}$ & 80 & 30 & $\mathrm{I}$ \\
\hline $\begin{array}{l}\text { 4. Posee conocimientos científico técnicos } \\
\text { suficientes }\end{array}$ & 80 & 75 & I \\
\hline $\begin{array}{l}\text { 5. Recibió capacitación para mejorar su } \\
\text { competencia }\end{array}$ & 80 & 70 & I \\
\hline $\begin{array}{l}\text { 6. Dispone de bibliografía y otros medios } \\
\text { para su actualización científica }\end{array}$ & 80 & 23 & I \\
\hline $\begin{array}{l}\text { 7. La actividad lo estimula a su auto } \\
\text { preparación }\end{array}$ & 80 & 48 & I \\
\hline $\begin{array}{l}\text { 8. Dispone de los recursos materiales } \\
\text { necesarios para realizar adecuado } \\
\text { desempeño }\end{array}$ & 80 & 65 & $\mathrm{I}$ \\
\hline $\begin{array}{l}\text { 9. Recibe apoyo de la administración para } \\
\text { resolver problemas de servicio }\end{array}$ & 80 & 59 & I \\
\hline 10. Recibe supervisiones a su trabajo & 80 & 70 & I \\
\hline $\begin{array}{l}\text { 11. Recibe apoyo necesario del MINED } \\
\text { para desarrollar su trabajo }\end{array}$ & 80 & 70 & I \\
\hline $\begin{array}{l}\text { 12.Recibe estímulos por cumplir o sobre } \\
\text { cumplir funciones laborales }\end{array}$ & 80 & 28 & I \\
\hline 13. Investiga actualmente & 80 & 20 & I \\
\hline
\end{tabular}

Nota Aclaratoria: A - Adecuado I - Inadecuado 
Aspecto importante a evaluar fue el nivel de satisfacción de los estomatólogo y licenciados en atención estomatológico con los servicios brindados, al evaluar estos criterios (tabla 1) obtuvimos que la satisfacción de los proveedores es inadecuada, de 13 criterios evaluados, solo el 7,7\% de los criterios resultó adecuado y 12 criterios resultaron inadecuados para un 92,3\%, reveló que a pesar de que el criterio: le gusta su trabajo superó el estándar establecido hubo una marcada insatisfacción laboral por no sentirse motivados para investigar.

Uno de los elementos básicos de la calidad de la vida laboral es la satisfacción con el trabajo. En organizaciones, como el servicio de salud, cuya consecuencia de objetivos, depende en buena medida de la voluntad de cooperar y el compromiso con el trabajo de los profesionales que lo integran es precisamente importante que encuentren elementos de satisfacción en el desempeño de su trabajo. ${ }^{12}$

Estos resultados no se corresponden con los obtenidos por el Dr. Castro ya que los profesionales se encontraban satisfechos en su estudio. ${ }^{12}$

Los criterios que demuestran mayor insatisfacción entre los profesionales de la salud se refieren a la bibliografía disponible (23\%) y a la preparación científico-técnica para su desempeño (30 \%), al no recibir cursos de post grado que apoyen la labor realizada tanto curativa como preventiva, lo que mejoraría la competencia y desempeño profesional. Las Licenciadas no se encuentran motivadas, al no poder realizar las actividades comprendidas en sus estudios, por dificultades administrativas.

Coincidimos en este aspecto con los resultados obtenidos por la Dra. Leyva, Hernández Arencibia y Cámbara Rondón en sus estudios de calidad realizados.

Vale la pena detenerse a puntualizar que no podemos tener un servicio de calidad total si los proveedores que ofrecen el mismo no están satisfechos. No resulta posible generar respuestas rápidas, supervisar y controlar su propia actuación, sí el trabajador no siente la actividad como algo muy propio de él. De ahí que la calidad total tenga que comenzar por una visión de lo que sucede hacia dentro, para poder predecir lo que sucede hacia fuera. ${ }^{13}$ 
El no contar con los materiales necesarios para la realización del trabajo trae como consecuencia desmotivación, al no poder efectuar los tratamientos requeridos en cada caso, lo que atenta negativamente para lograr una atención con la calidad óptima.

De acuerdo a la evaluación realizada, planteamos que la satisfacción de los proveedores es inadecuada con respecto al servicio que prestan, al obtenerse un 92,3\% de criterios con esta calificación.

La satisfacción del usuario representa el grado en que la atención brindada satisface sus expectativas, esta dimensión no guarda, necesariamente, una directa relación con el nivel de calidad científico técnica de la atención y viceversa ${ }^{11}$

Tabla 2. Tabla de evaluación de la satisfacción de los usuarios con el servicio recibido.

\begin{tabular}{|l|c|c|c|}
\hline Criterios & $\begin{array}{c}\text { Estándar } \\
\%\end{array}$ & $\begin{array}{c}\text { Resultados } \\
\%\end{array}$ & Evaluación \\
\hline $\begin{array}{l}\text { 1.Considera usted que los conocimientos } \\
\text { que se han brindado a su hijo sobre el } \\
\text { mantenimiento de la salud bucal en la } \\
\text { escuela han sido adecuados }\end{array}$ & 95 & A \\
\hline $\begin{array}{l}\text { 2. Cómo evalúa usted el trato del personal } \\
\text { estomatológico que brinda atención en la } \\
\text { escuela. }\end{array}$ & 95 & A \\
\hline $\begin{array}{l}\text { 3. Considera la atención estomatológica } \\
\text { que ha recibido su hijo en la escuela } \\
\text { satisfactoria. }\end{array}$ & 85 & A \\
\hline $\begin{array}{l}\text { 4. Está satisfecho con las actividades de } \\
\text { prevención que se le han realizado a su hijo } \\
\text { en la escuela: enjuagatorios de flúor } \\
\text { (buchadita), aplicación de laca flúor } \\
\text { (pastica). }\end{array}$ & 90 & 95 & A \\
\hline
\end{tabular}


Para evaluar (tabla 2), el nivel de satisfacción de los usuarios con la atención recibida se tuvieron en cuenta 4 criterios, resultando la evaluación adecuada, al alcanzar el 100\% de los criterios el estándar establecido.

Los resultados de este estudio se corresponden con los resultados obtenidos por el Dr. Castro en el cual las evaluaciones finales fueron satisfactorias. (Trabajo para Optar por el Título de Máster en Urgencia Estomatológica 2008).

Es el paciente la principal razón de una entidad o servicio y su satisfacción un instrumento para medir la eficacia del mismo, el que este se muestre satisfecho puede conducir al falso criterio de haberse logrado la calidad esperada en la atención que se brinda.

La satisfacción del paciente, representa la evaluación que hace acerca de la calidad de la atención y está determinada por la percepción que el mismo haga de la medida en que satisfagan sus expectativas de la atención de una buena calidad.

Los pacientes por lo general tienen un conocimiento incompleto de las bases científico - técnicas de la atención estomatológica por lo que sus juicios en estos aspectos puede no ser válido; en algunos casos esperan y exigen cosas que sería incorrecto que el profesional proporcionara; no obstante estas limitaciones no restan validez a la satisfacción del paciente como una medida de la calidad de la atención. ${ }^{14}$

El otro elemento evaluado fue la satisfacción de los proveedores y la población, en el que la totalidad de los criterios obtuvieron resultados inadecuados, debido en especial a los aspectos del salario y el disgusto por el trabajo en el terreno, que fueron los más cercanos al estándar. El personal evaluado argumentó que la alimentación no poseía la que el salario, a pesar de incremento en estos últimos tiempos lo consideran insuficiente; por otro lado, el disgusto por el trabajo en el terreno se atribuyó a los constantes controles y la fiscalización de que eran objeto. Otra serie realizada en La Habana también obtuvo resultados similares.

Según Roger Ruiz-Moral, el aspecto más y tal vez mejor estudiado de los diferentes componentes de la relación médico-paciente (RMP) es el comunicativo. Determinados estilos de comunicación, especialmente el denominado "estilo centrado en el paciente" se han asociado 
positivamente con la satisfacción y otros resultados de la consulta. Sin embargo, las mediciones de la percepción de la calidad comunicativa de la RMP están a menudo limitadas por la tendencia a dar respuestas aquiescentes, es decir la tendencia de los pacientes a mostrarse satisfechos sin tener en cuenta el contenido o incluso aún con interacciones comunicativamente deficientes. En nuestro estudio los clientes opinaron que la comunicación por parte del proveedor fue adecuada, ya que obtuvieron respuestas a su problemática. ${ }^{8}$

Por todas estas razones pudo concluirse que el nivel de satisfacción de los proveedores fue inadecuado y usuario fue adecuada.

\section{Referencias bibliográficas}

1. Massip Pérez C. La evaluación de la satisfacción en salud: un reto a la calidad. Rev Cubana Salud Pública 2008 [citado 11 enero 2016]; 34(4). Disponible en: http://scielo.sld.cu/scielo.php?script=sci_arttext\&pid=S0864$\underline{34662008000400013 \& \operatorname{lng}=\mathrm{es}}$

2. Jiménez Cangas 1. Metodología para la evaluación de la calidad en instituciones de atención primaria de salud. Rev Cubana Salud Pública. 1996 [citado 11 enero 2016]; 22(1). Disponible en: http://scielo.sld.cu/scielo.php?script=sci_arttext\&pid=S0864$\underline{34661996000100008 \& \operatorname{lng}=\mathrm{es}}$

3. Laurenza M, Marracino C. Experiencia en la aplicación de indicadores de calidad en Hospitales Públicos de la República Argentina 2011. [citado 11 enero 2016]. Disponible en: http://www.itaes.org.ar/dwnl/Indicadores-de-Calidad2.pdf

4. Garrido Latorre F, López Moreno S. Evaluación de los programas y servicios de salud en México. Salud Pública Méx 2011. [citado 11 oct 2016]; 53 (supl 4). p. 399-401. Disponible en: http://www.scielo.org.mx/scielo.php?script=sci_arttext\&pid=S003636342011001000002

5. OMS, OPS. Ministerio de Salud. Evaluación de calidad. Una guía de los servicios de salud amigables para los adolescentes 2009. [citado 11 enero 2016]. Disponible en: 
http://publicaciones.ops.org.ar/publicaciones/publicaciones\%20virtuales/EvaluacionCalid adVirtual /pdfs/GuiaAdolescentes.pdf

6. Sosa Rosales M. González Ramos R M. Gil Ojeda E. Programa de mejora continua de La calidad de la atención Estomatológica y la Satisfacción de la población y Los prestadores. 2002. Ciudad de la Habana, CUBA. [citado 11 enero 2016]. Disponible en: http://files.sld.cu/sida/files/2012/01/prog-calidadatencion-estomatol.pdf

7. Jacob Ak. Rebuilding an enduring truest in medicine. A globalmandate Circulation. Presidential. Address American Heart Association Scientific Sessions. 2005; 111 (25). p. 3494-8.

8. Morales P K. Dimensiones involucradas en la evaluación de la calidad. 2014 [citado 11 enero 2016].

Disponible en: repositorio.uchile.cl/bitstream/.../Vergara\%20Henríquez\%20María.pdf?

9. Ruiz-Moral, R. Satisfacción de los pacientes con la comunicación en las consultas de medicina de familia: comparación de tres métodos para investigar necesidades no satisfechas. Rev. Esp. Salud Publica 2011; 85(3). p.315-322. [citado 11 enero 2016]. Disponible en: <http://scielo.isciii.es/scielo.php?script=sci_arttext\&pid=S1135$57272011000300010 \& \operatorname{lng}=$ es\&nrm=iso>.

10. Garrigó Abreu. Guías prácticas clínica de caries dental. En: Sosa Rosales M. Guías Prácticas de Estomatología. 2ed. La Habana. Editorial Ciencias Médicas, 2007. p. 23-5

11. Agra Varela, García Baró S, Magrinya Rull P .Manual de calidad en Atención Primaria de Salud .Madrid: Imprenta de la Comunidad de Madrid; 1994. p. 66 - 171.

12. Hechavarria M. N, Cairo Soler C. Administración y Gestión de los Servicios de la Información. La Habana: Editorial Ciencias Médica; 2004. p. 193-99.

13. Suárez LN. Marketing de Salud: Calidad de los servicios. Competencia. Determinantes de la calidad. Calidad total. En: Fernández CE, Castell-Florit SP. Sistema de Capacitación 
Gerencial en Salud: Selección de temas. La Habana. MINSAP; 2004 Tomo 2. p. 222228.

14. Zalasvsky AM, Beaulie ND, Landon BE, Cleary PD. Dimensions of consumer -assesserd quality of Medicare managed - care heald plans. Med Care 2000; 38(2). p. 162-74. 